\title{
Review
}

\section{RNA back and forth: Looking through ribozyme and viroid motifs}

\author{
Marie-Christine Maurel ${ }^{1 *(D)}$, Fabrice Leclerc ${ }^{2}{ }^{(0}$, Jacques Vergne ${ }^{1}$ and Giuseppe Zaccai ${ }^{3}(\mathbb{C}$ \\ 1 Sorbonne Université, Museum National d'Histoire Naturelle, CNRS MNHN UMR 7205 , Institut de \\ Systématique, Evolution, Biodiversité, ISYEB, F-75005 Paris, France; \\ marie-christine.maurel@sorbonne-universite.fr \\ 2 Institute for Integrative Biology of the Cell (I2BC), CNRS, CEA, Universite Paris Sud, Gif- sur-Yvette, \\ F-91198, France; fabrice.leclerc@i2bc.paris-saclay.fr \\ 3 Institut de Biologie Structurale CNRS-CEA-UGA, F-380447 Grenoble, France, and Institut Laue Langevin, 71 \\ Avenue des Martyrs, F-38042 Grenoble, France; zaccai@ill.fr \\ * Correspondence: marie-christine.maurel@sorbonne-universite.fr; Tel.: +33-01-40-33-79-81
}

\begin{abstract}
Current cellular facts allow us to follow the link from chemical to biochemical metabolites, from the ancient to the modern world. In this context, the "RNA world" hypothesis proposes that early in the evolution of life, the ribozyme was responsible for the storage and transfer of genetic information and for the catalysis of biochemical reactions. Accordingly, the hammerhead ribozyme (HHR) and the hairpin ribozyme, belong to a family of endonucleolytic RNAs performing self-cleavage that might occur during replication. Furthermore, regarding the ultraconserved occurrence of HHR in several genomes of modern organisms (from mammals to small parasites and elsewhere), these small ribozymes have been regarded as living fossils of a primitive RNA world. They fold into 3D structures that generally require long-range intramolecular interactions to adopt the catalytically active conformation under specific physicochemical conditions. By studying viroids as plausible remains of ancient RNA, we recently demonstrated that they replicate in non-specific hosts, emphasizing their adaptability to different environments, which enhanced their survival probability over the ages. All these results exemplify ubiquitously features of life. Those are the versatility and efficiency of small RNAs, viroids and ribozymes, as well as their diversity and adaptability to various extreme conditions. All these traits must have originated in early life to generate novel RNA populations.
\end{abstract}

Keywords: RNA world; viroid; ribozyme; origins of life

\section{Introduction}

\subsection{The RNA World}

We know today that RNA is present in all living organisms, in which it performs a variety of structural and metabolic functions. A truly modern "RNA world" exists in each cell, with RNAs in various forms, short and long fragments, single and double-stranded, endowed with multiple roles, informational, catalytic, structural and regulatory ( $98 \%$ of the human genome is non-coding, transcribed almost entirely, acting as sensors, traveling between organisms, etc). Certain RNA molecules are even capable of carrying out several of these functions.

The "RNA World" hypothesis as an early step in the history of life was so-named by Walter Gilbert [1] after being proposed in the preceding decades by Carl Woese, Francis Crick and Leslie Orgel [2-4]. Strong support for the hypothesis was provided by the discovery of small endonucleolytic ribozymes. These are hairpin and hammerhead catalytic motifs within viruses, viroids and satellite RNAs [5,6], which perform reversible self-cleavage reactions involved in processing and replication of small circular RNA species $[7,8]$. The RNA World hypothesis raises important questions. Could RNA have existed alone? Is there a «fossil» trail of ancient RNAs ? 


\subsection{Viroids and Ribozymes}

Viroids are the smallest plant pathogens. From 300 to 500 ribonucleotides long, the single-stranded RNA molecules can pair, form loops and bulges. They were discovered in 1971 by Theodor Diener [9] following the observation of potato tubercle disease, which was first attributed to bacterial infection, then to a virus, then to viroids. Viroids are absolutely «free» in nature. As opposed to viruses, they are composed of free RNA without envelope or capsid and do not code for any protein. Some viroid structures are similar to those of other RNAs from which they might have emerged; for example, ASBVd and transfer RNA [10,11] or small plant circular RNA [12]. Viroids are circular, rod-like or flexible, the existence of several different motifs enhancing the probability of survival. They replicate in either the cell nucleus or chloroplast, suggesting diversity and adaptability to different environments. Two viroid families are known today, the Pospiviroidae including 5 genera (pospiviroids, hostuviroids, cocadviroids, apscaviroids and coleviroids) and 27 species, and the Avsunviroidae with three genera (avsunviroids, pelamoviroides et elaviroides) and 4 species. Pospiviroidae replicates in the cell nucleus by the asymmetric rolling circle mechanism and in chloroplasts for Avsunviroidae by the symmetrical rolling circle mechanism which implicates a hammerhead ribozyme (HHR). In this symmetric variant of the rolling circle mechanism, the monomeric $(+)$ circular RNA serves as the template for an RNA polymerase that replication leads to a linear (-) oligomer. This RNA, self-cleaves through a hammerhead ribozyme from RNA itself. The monomeric (-) linear RNA is then ligated to the circular form that serves as the template for synthesis of the linear $(+)$ oligomer, which after self-cleavage and circularization gives the final product that is the monomeric (+) circular RNA [13-15]. The mechanistic aspects of the cleavage reaction in the hammerhead motifs is as follows: initiation by a nucleophilic attack of the 2'-hydroxyl group on the adjacent phosphorus. The reaction mechanism is SN2, with a trigonal bipyramidal transition state. The departure of the 5'-oxyanion leaves a 2'-3' cyclic phosphate. The transesterification reaction requires divalent metal ions and is accelerated by general acid?base catalysis and by conformational effects facilitating the formation of the in-line geometry. The ligation is the exact reverse of the cleavage reaction [16].

Considering the absence of envelop, the fact that viroids are non-coding and the existence of ribozymes, it is tempting to consider them as vestiges of ancient RNA as Diener proposed as early as 1989. One supplementary supporting argument is their adaptability to other hosts than plants, non-photosynthetic Eukarya and Bacteria. This capability may help to elucidate several unexplained symptoms in other species than plants.

\subsection{The Avocado Sun Blotch Viroid (ASBVd)}

We have studied the viroid model: Avocado Sun Blotch Viroid (ASBVd), one of the smallest rod-like viroids ( $247 \mathrm{nts}$ ) including a hammerhead ribozyme (HHR) of 42 nucleotides. In vivo, both complementary strands $(+)$ and (-) can be used as template for replication once the first replication cycle completed from the infecting single-stranded RNA: ASBVd (+) conventionally designed for the most abundant infectious RNA polarity can be found in different concatenated multimeric forms up to octamers, while ASBVd (-) is just present as a monomer or dimer with a more efficient cleavage activity [14,17]. On native gel and thermal gradient gel electrophoresis (TGGE) [18] one major profile was observed for ASBVd (-) at all temperatures, while ASBVd (+) adopts alternative folds at lower temperatures. The derivative absorbance $(\mathrm{d} \triangle \mathrm{A} 260 / \mathrm{dT})$ of the melting profiles (Fig. 1) shows two transitions for ASBVd (+) and one for ASBVd (-) [19]. We conclude of 2 non-symmetrical polarities (+) and (-) with different structures and different catalytic activities. 
A

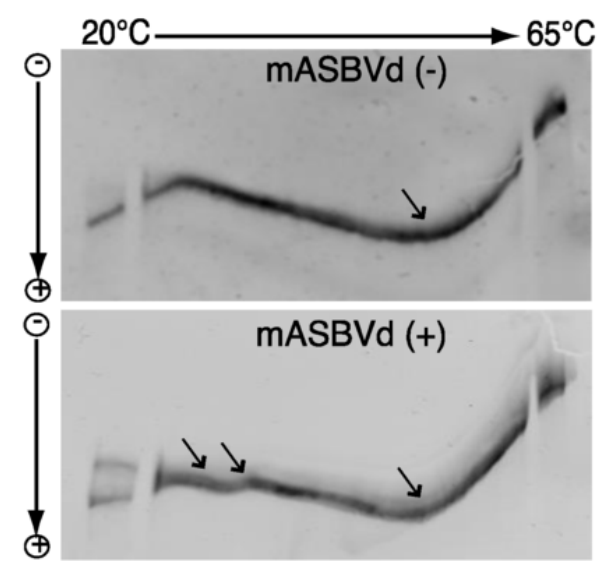

C

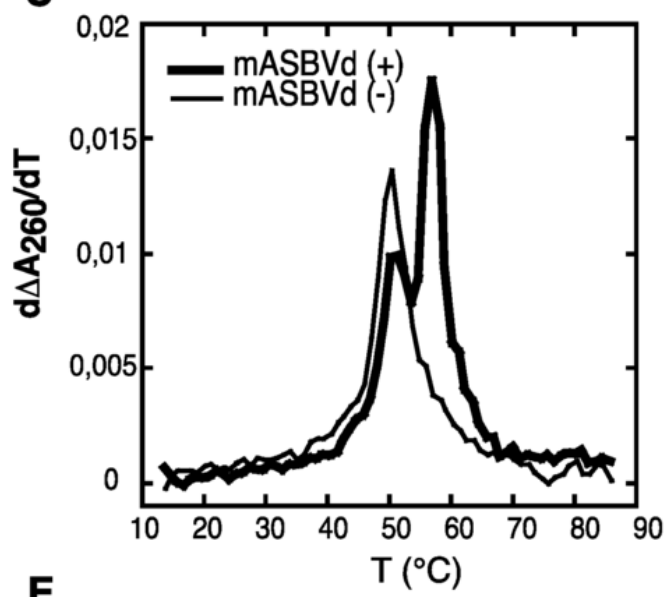

E

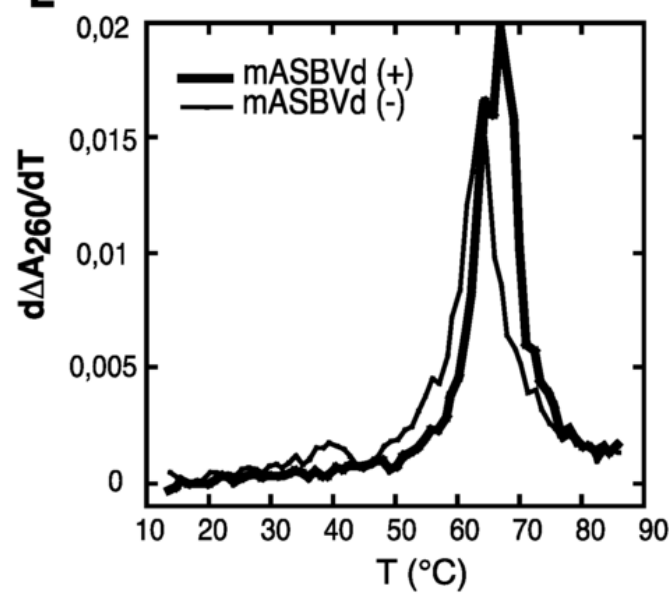

B

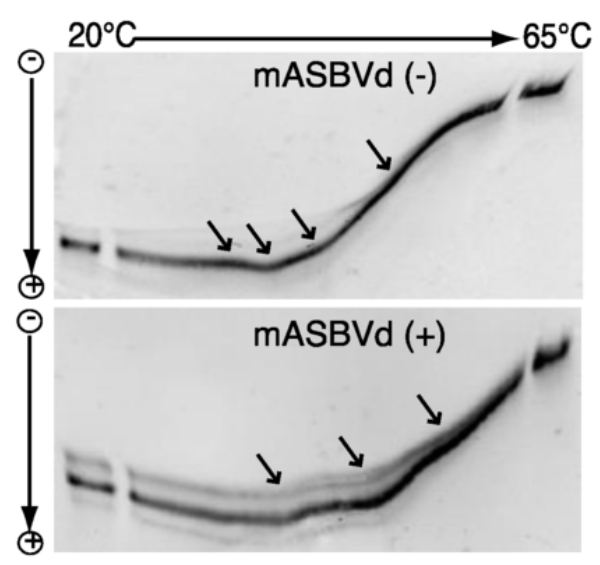

D

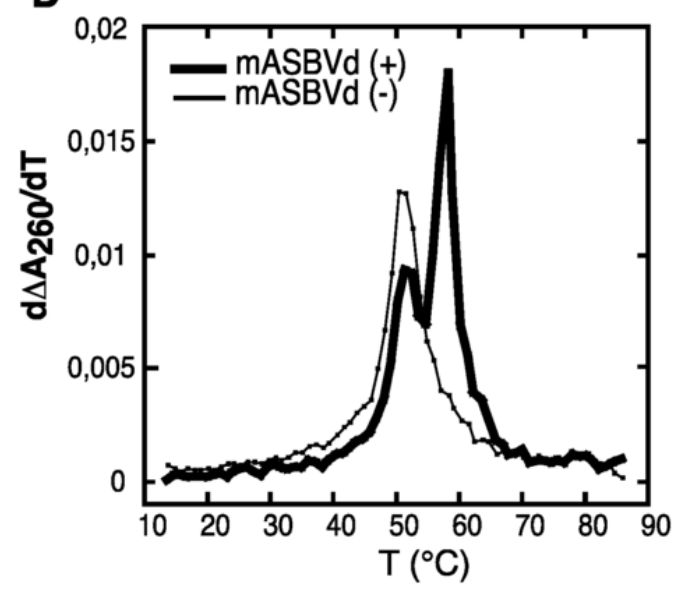

Figure 1. TGGE and melting curves of mASBVd (+) and mASBVd (-) transcripts.(A) TGGE analysis performed by native gel electrophoresis $8 \%$ PAGE in 0.2 TBE Buffer. (B) TGGE analysis performed by native gel electrophoresis $8 \%$ PAGE in 0.2 TBE Buffer containing $20 \mathrm{mM}$ magnesium acetate. Migration was monitored between $20^{\circ} \mathrm{C}$ and $65^{\circ} \mathrm{C}$. The arrows denote the transition temperatures. Derivative absorbance melting profile were determined either in $150 \mathrm{mM} \mathrm{KCL}$ and $10 \mathrm{mM}$ sodium cacodylate $(\mathrm{pH}$ 7.2). (C) or with in addition, $100 \mathrm{mM} \mathrm{MgCl} 2$ (D) or $1 \mathrm{M} \mathrm{NaCl}$ (E) The first derivative profiles are shown with a thick curve for mASBVd (+) and a thin curve for mASBVd (-). From Delan-Forino et al. [19]. 


\section{Results}

\subsection{Viroid structures and interactions revealed by Small Angle Neutron Scattering combined with Modelling}

Small angle neutron scattering (SANS) is a well-adapted method to study fragile nucleic acid structures and interactions in solution $[20,21]$. The sample solution of the order of $\mathrm{mg} / \mathrm{mL}$ is examined in a quartz cell, where solvent conditions and/or temperature can be easily changed, in-beam. There is no radiation damage to the sample even when irradiated for a long time at high temperature. SANS data in the Guinier approximation are interpreted, on an absolute scale, in terms of low resolution mean structural parameters for the particles in the solution [20,21]. From $I(0)$, the forward scattered intensity, is obtained the number-average molecular weight of the particles (distinguishing monomers from dimers, for example); from the angular dependence of the intensity, are obtained particle radii of gyration and cross-sectional radii of gyration for rod-like configurations.

Bi-molecular RNA interactions such as linear-linear, loop-loop, loop-linear or kissing interactions are important in the control of biological activity, and hairpin loops present rich potential for establishing both intra- and intermolecular interactions through standard Watson-Crick base pairing or non-canonical interactions. In a previous study, we applied SANS with native gel electrophoresis and analytical centrifugation to the structures and self-interactions of two 85 base adenine-dependent hairpin ribozymes (ADHR1 and ADHR2) [22]. Similar results were obtained for the two. At room temperature, the ribozymes self-associated in structures with a cross-section corresponding to two double strands side-by-side. Non-covalent dimers predominate at low concentration $(\sim 0.1 \mathrm{mg} / \mathrm{ml})$; they associate into longer rods, with increasing concentration $(\sim 1 \mathrm{mg} / \mathrm{ml})$. Above $65^{\circ} \mathrm{C}$, the structures dissociated into compact monomers, with a radius of gyration similar to that of tRNA (about 70 bases). Only monomers were active for catalysis, suggesting that dimer formation, probably by preventing correct loop docking, could act as an inhibition mechanism for the regulation of hairpin ribozyme catalysis.

The SANS approach developed in the ADHR study was applied to ASBVd (-) and its derived 79-nt HHR (-). It revealed a reversible temperature-dependent dimer association in both molecules. In Fig. 2A, I(0) values (normalized to 1 for the monomer) are seen to oscillate in the region between 1 (100\% monomers $\mathrm{M}$, at high temperature) and $2(100 \%$ dimers $\mathrm{D}$, at low temperature). HHR $(-) / \mathrm{Mg}$ (in presence of $\mathrm{Mg++}$ ) displays a striking temperature driven oscillatory behavior, between dimer-dominated scattering at low temperature and monomer-dominated scattering at the higher temperature. The values for ASBVd (-) (Fig. 2B) indicated a reversible low level of particle association at low temperature (accounted for by about $5 \%$ of the particles forming dimers) with full dissociation to monomers at the higher temperature, both in presence and absence of $\mathrm{Mg}++$. To explore the functional relevance of the SANS observations, the Arrhenius plot calculated from the rate of HHR (-)/Mg dimer dissociation as a function of temperature was compared to a similar plot calculated for catalytic activity of the same ribozyme published previouslyÂ (El-Murr 2012) (Fig. 2C). The two plots are strikingly similar, showing a break at about $27^{\circ} \mathrm{C}$ between regimes of activation energy $7 \pm 1 \mathrm{kcal} / \mathrm{mol}$ and $25 \pm 5$ $\mathrm{kcal} / \mathrm{mol}$, at higher and lower temperatures, respectively, suggesting that the rate-determining step corresponds to the dimer/monomer transition, with dimer dissociation correlated with higher activity. 
A
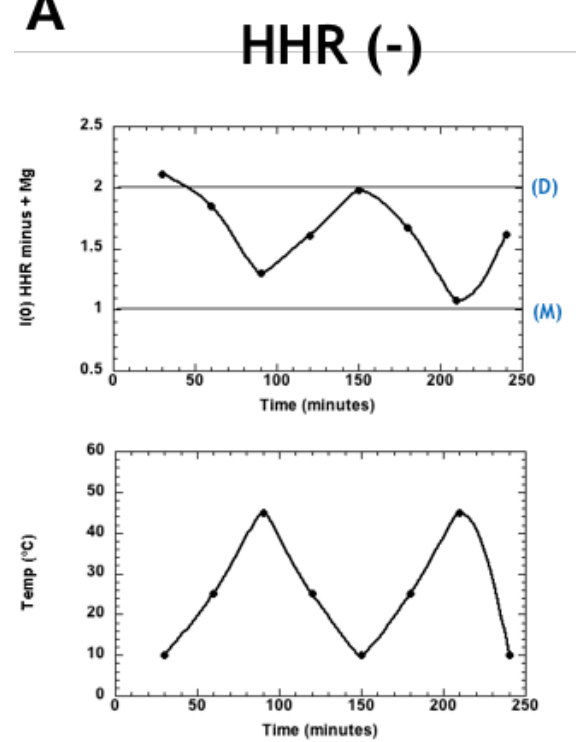

B
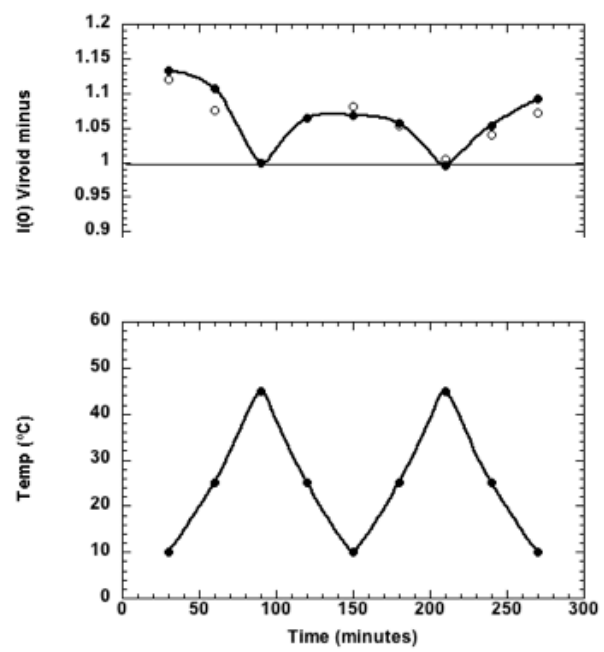

C

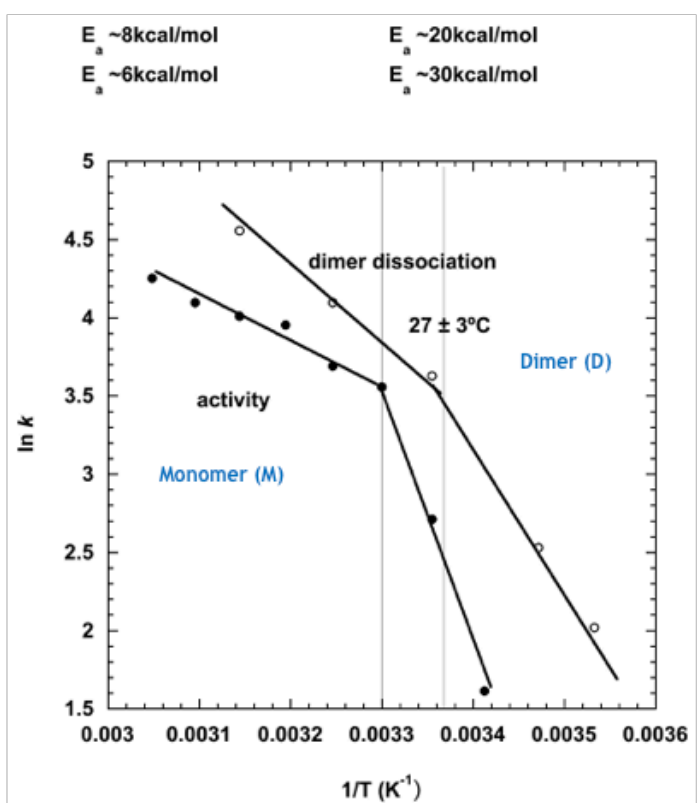

Figure 2. (A) Normalised forward scattered SANS intensity for HHR (-) in presence of $\mathrm{Mg}++$ at the corresponding temperature on the plot below. The values at 1.00 and 2.00 correspond to the intensity expected for the monomer (M) and dimer (D), respectively. (B) Normalised forward scattered SANS intensity for ASBVd (-) in presence (filled circles) and absence (open circles) of $\mathrm{Mg}++$, at the corresponding temperature on the plot below. The data indicate a small amount of dimer formation at the lower temperature. (C) Arrhenius plots of HHR (-)/Mg rate of dimer dissociation (open circles, calculated from (A), and the ribozyme's catalytic activity (filled circles), revealing parallel behaviour. Modified from Leclerc et al. [23]. 
2D and 3D modeling of monomeric and dimeric HHR (-) suggested that the intermolecular contacts stabilizing the dimer (between HI and HII domains) compete with the intramolecular ones stabilizing the active conformation of the full-length HHR required for an efficient self-cleavage (Fig. 3). Similar competing intra- and intermolecular contacts were proposed in ASBVd (-) though with a remoter region from an extension of the HI domain (Fig. 3). The structural basis for the lower catalytic activity in the HHR dimer is due to a competition between the intermolecular contacts responsible for the self-association of the two monomers and the long-range intramolecular contacts that stabilize the active conformation of the HHR monomer. In the dimer complex (Fig. 3), the first monomer is expected to be fully active since all the intramolecular contacts are preserved for the RNA to fold into its catalytically active conformation. On the other hand, these intramolecular contacts are abolished in the second monomer because the corresponding residues are involved in the monomer-monomer interaction (Fig. 3A). The 3'-end from the first monomer interacts with the loop region of the HII domain from the second monomer, thus preventing the second monomer to adopt its active conformation necessary for an efficient catalysis (Fig. 3B). This loop region is remodeled during the self-association so that it can pair to the single-stranded 3 '-end of the first monomer. The resulting paired region corresponding to a half helix turn, and the residues involved in the intramolecular contacts preserved in the first monomer or lost in the second monomer are shown in details in Fig. 3C. The dimer adopts a conformation where only the first monomer can be fully stabilized in the active conformation, the active site from the second monomer being disorganized due to the intermolecular contacts formed during the self-association [23] (Fig. 3D).

\subsection{Replication of ASBVd in other kingdoms}

It remains to be determined whether or not ASBVd can replicate in other types of plastids (e.g. proplastids, amyloplasts, etioplasts, and chromoplasts) and in other species than plants. Following this line of thought we showed an efficient cleavage of ASBVd by the HHR and followed the transcription/replication as well as circularisation of the viroid in the yeast $S$. cerevisiae. The process was followed for 25 generations that is the persistence of the viroid in S. cerevisiae, showing unsuspected functional diversity and adaptability and confirming that the ribozyme can fulfill the cleavage/ligation reaction during the rolling circle replication process in a non-plant host [24].

The RNAs were extracted at different times from 0 to $50 \mathrm{~h}$ and then analyzed by Northern blotting (Fig. 4A-B). After $50 \mathrm{~h}$ corresponding to about 25 generations, both polarities of $\mathrm{mASBVd}$ were still detected (Fig. 4A-B, lanes 8 and 14). These results strongly suggest that the plasmid transcription is not mandatory for the maintenance of mASBVd via RNA-RNA replication in yeast, at least during 25 generations. We concluded on the functional diversity and unsuspected adaptability to a non-plant host.

Because ASBVd replicates in plant chloroplasts which have their ancestry in cyanobacteria, via endosymbiosis [25], we asked ourselves if one can detect the presence of viroids in the Bacterial domain, in particular, in cyanobacteria. Anabaena (Anabaena sp. PCC7120) is ̂̂ a filamentous cyanobacterium that is a photosynthetic prokaryote (blue-green algae). We detected (-) and (+) ASBVd in Anabaena, that is linear replicate (+) was detected by riboprobe (-) when RNAs/ pRLASBVd(-) was loaded as well as linear replicate (-) detected by riboprobe (+) when RNA/pRLASBVd(+) was loaded [26] (Fig. 5).

The replication of ASBVd in Anabaena had no effect on the physiology of this filamentous cyanobacterium. Replication of the viroid in S. cerevisiae has also been shown to be asymptomatic [24]. Together, these results raise the question on whether or not various non-infectious RNAs may exist across the Bacteria, Archaea, Eukarya realms where they would replicate via an RNA-RNA mechanism. If so, what are the evolutionary forces that maintained this "RNA World" in parallel to the "DNA World" ? 
A

B

C

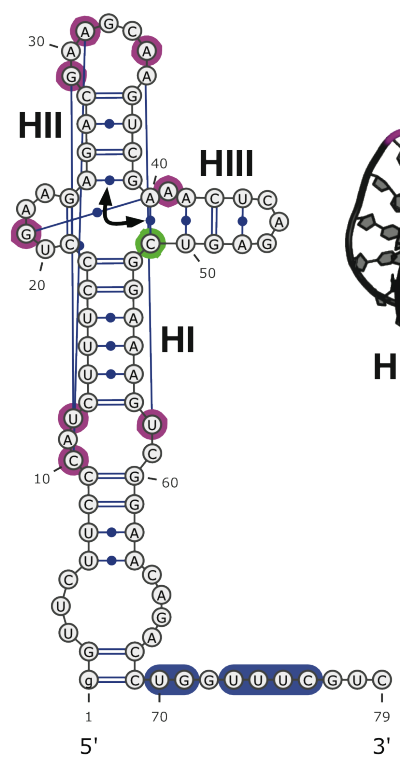

HIII

D
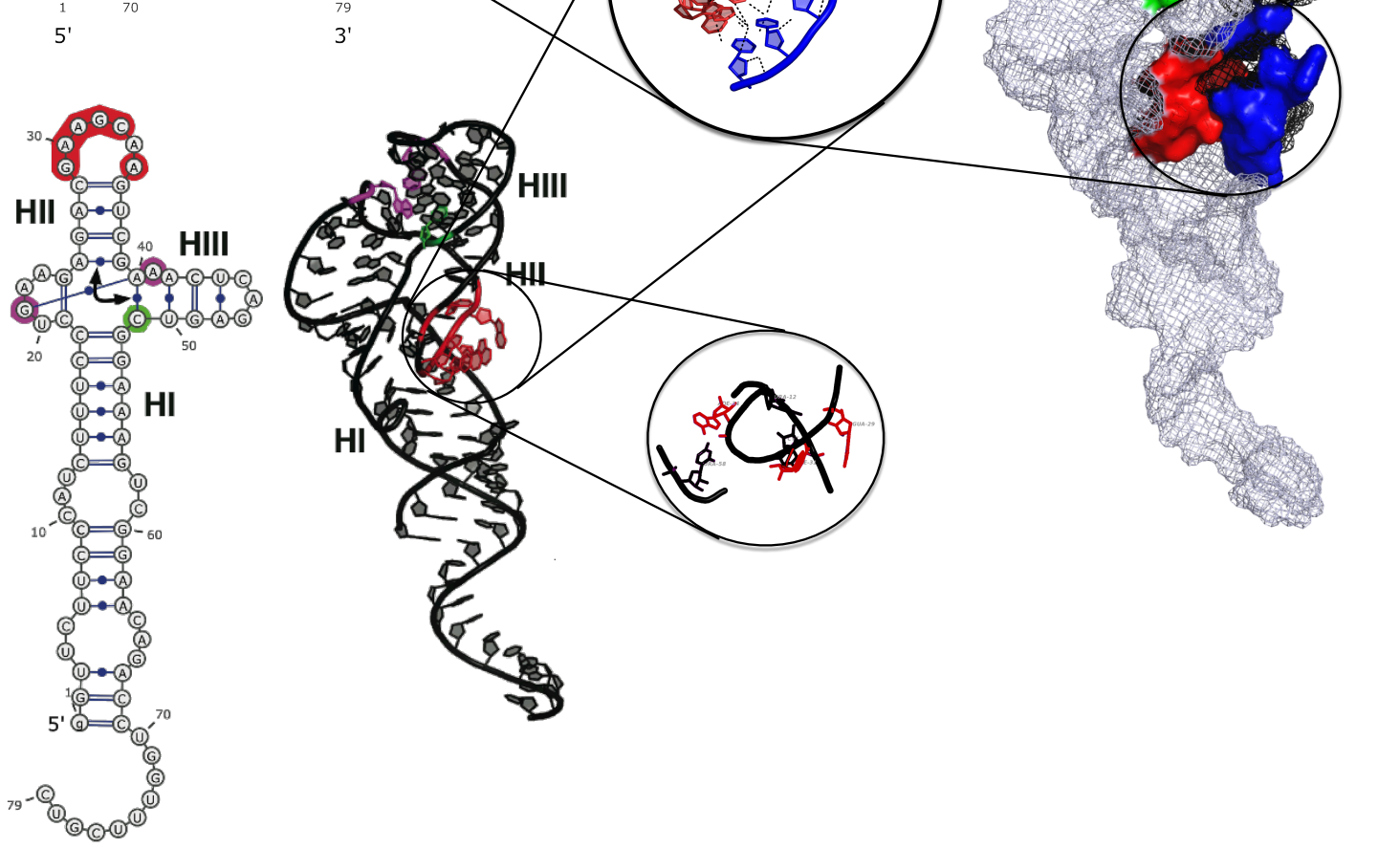

Figure 3. 3D Model of self-association for the hammerhead ribozyme (HHR) from ASBVd(-).(A) 2D structures of the two monomers as they are in the HHR dimer. (B) 3D structures of the two monomers as they are in the HHR dimer. (C) Zoom in on the regions involved in the monomer-monomer interactions and in the 3D contacts within the first monomer (top). (D) 3D structure of the HHR dimer. The long-range intramolecular contacts are indicated in magenta and the cleavage site in green. The paired regions are blue (first monomer) and red (second monomer). Modified from Leclerc et al. [23]. 

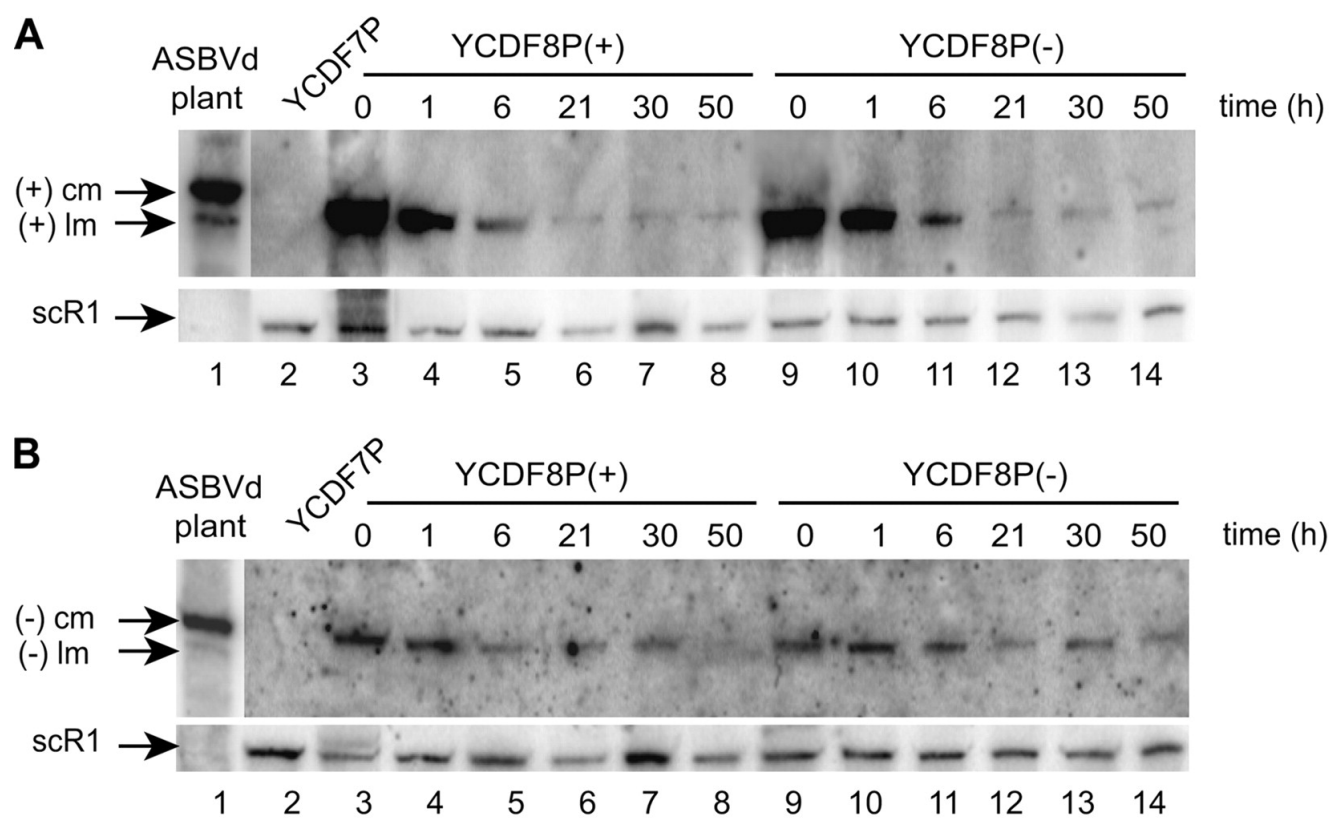

Figure 4. Northern blot analyses of the persistence of mASBVd in S. cerevisiae. (A) mASBVd (+) evidenced by riboprobe (-) and (B) mASBVd (-) evidenced by riboprobe $(+)(\mathrm{cm}$ : circular monomer; $\mathrm{lm}$ : linear monomer). (A-B) Total RNAs were extracted from the YCDF7P, YCDF8P(+), and YCDF8P(-) strains. From Delan-Forino et al. [24].

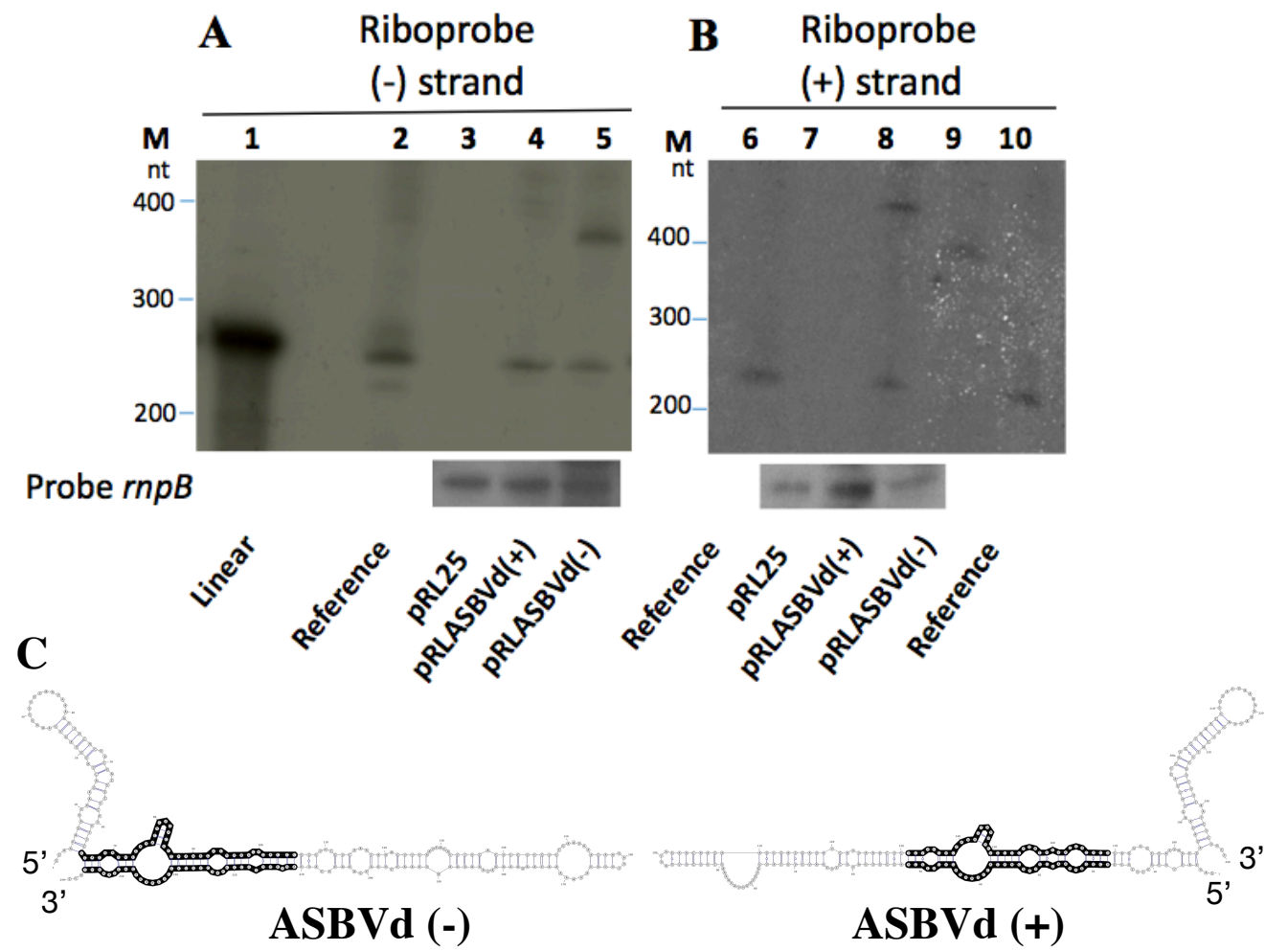

Figure 5. Analysis of ASBVd replication in the filamentous cyanobacteria Anabaena. RNAs extracted from Anabaena harboring either the empty pRL25 plasmid $(3,7)$, pRLASBVd $(-)(5,9)$ or pRLASBVd $(+)(4,8)$. Northern blot analysis by hybridization using riboprobes corresponding to the ASBVd strands of $(-)(\mathbf{A})$ or $(+)($ B) polarities. Lane 5: linear replicate $(+)$ detected by riboprobe (-) when RNAs/ pRLASBVd(-) was loaded. Lane 8: linear replicate (-) detected by riboprobe (+) when RNAs /pRLASBVd(+) was loaded. (C) The full length genome of ASBVd can fold into a 2D structure that preserves the HHR motif in both the (-) and (+) strands (left and right, respectively); the HHR motif of ASBVd (-) is more stable. Modified from Latifi et al. [26]. 


\section{Conclusions}

ASBVd exists in two non-symmetrical polarities (+) and (-) with different structures and catalytic activities. A joint thermodynamic analysis of structural and catalytic data indicated the rate-determining step corresponds to a dimer/monomer transition. Models suggest that the intermolecular contacts stabilizing the dimer (between HI and HII domains) in ASBVd (+) compete with the intramolecular ones stabilizing the active conformation of the full-length HHR required for efficient self-cleavage. Similar competing intra- and intermolecular contacts are proposed in ASBVd (-) though with a remoter region from an extension of the HI domain. In vivo, ASBVd (+) can be found in different concatenated multimeric forms up to octamers, while ASBVd (-) is just present as a monomer or dimer with a more efficient cleavage activity. Each polarity could play a distinct role during the viroid life cycle.

Adaptation to different hosts: ASBVd that normally replicates in the chloroplast of avocado, is also able to replicate in the cyanobacterium Anabaena. We have also shown that ASBVd replicates and persists in S. cerevisiae, a non-photosynthetic eukaryote. It is legitimate to speculate on extant viroids as descendants of "free-living" proviroids that invaded ancient cyanobacteria, which would later became endosymbionts, evolving in chloroplasts by usurping the biochemistry of their hosts. Finally, in a "viroids as living fossils" scenario [27], we must envisage that polymerase and ligase activities might have been lost by the viroid ancestors, since it has been shown that these two activities are possible in vitro. They were indeed discovered in artificially evolved RNA by SELEX, for instance, in the RNA-polymerase ribozyme (PDB ID: 3IVK) [28] and RNA ligase ribozyme (PDB ID:2OIU) [29].

Author Contributions: All the authors contributed to the writing and editing of the manuscript.

Funding: This research received no external funding.

Acknowledgments: We thank the CNES (Centre National d'Etudes Spatiales) for constant support for the studies on the origins of life.

Conflicts of Interest: The authors declare no conflict of interest.

\section{Abbreviations}

The following abbreviations are used in this manuscript:

ASBVd (Avocado Sun Blotch Viroid),

HHR (Hammerhead ribozyme),

TGGE (Temperature gradient gel electrophoresis),

ADHR (adenine-dependent hairpin ribozyme),

SANS (small-angle neutron scattering).

1. Gilbert, W. Origin of life: The RNA world. Nature 1986, 319, 618-618. doi:10.1038/319618a0.

2. Woese, C.R. On the evolution of the genetic code. Proceedings of the National Academy of Sciences 1965, 54, 1546-1552. doi:10.1073/pnas.54.6.1546.

3. Crick, F. The origin of the genetic code. Journal of Molecular Biology 1968, 38, 367-379. doi:10.1016/0022-2836(68)90392-6.

4. Orgel, L. Evolution of the genetic apparatus. Journal of Molecular Biology 1968, 38, $381-393$. doi:10.1016/0022-2836(68)90393-8.

5. Bajaj, P.; Steger, G.; Hammann, C. Sequence elements outside the catalytic core of natural hairpin ribozymes modulate the reactions differentially. Biological Chemistry 2011, 392. doi:10.1515/bc.2011.071.

6. Flores, R.; Serra, P.; Minoia, S.; Serio, F.D.; Navarro, B. Viroids: From Genotype to Phenotype Just Relying on RNA Sequence and Structural Motifs. Frontiers in Microbiology 2012, 3. doi:10.3389/fmicb.2012.00217.

7. Bajaj, P.; Hammann, C. Characterization of Hairpin Ribozyme Reactions. In Methods in Molecular Biology; Humana Press, 2013; pp. 97-111. doi:10.1007/978-1-62703-730-3_8. 
8. Jimenez, R.M.; Polanco, J.A.; Lupták, A. Chemistry and Biology of Self-Cleaving Ribozymes. Trends in Biochemical Sciences 2015, 40, 648-661. doi:10.1016/j.tibs.2015.09.001.

9. Diener, T. Potato spindle tuber "virus". Virology 1971, 45, 411-428. doi:10.1016/0042-6822(71)90342-4.

10. Ohnishi, K.; Hokari, S.; Shutou, H.; Ohshima, M.; Furuichi, N.; Goda, M. Origin of most primitive mRNAs and genetic codes via interactions between primitive tRNA ribozymes. Genome Inform 2002, 13, 71-81.

11. Ohnishi, K.; Ohshima, M.; Furuichi, N. Evolution from possible primitive tRNA-viroids to early poly-tRNA-derived mRNAs: a new approach from the poly-tRNA theory. Genome Inform 2005, 16, 94-103.

12. Hernández, C.; Daròs, J.; Elena, S.; Moya, A.; Flores, R. The strands of both polarities of a small circular RNA from carnation self-cleave in vitro through alternative double- and single-hammerhead structures. Nucleic Acids Res 1992, 20, 6323-9.

13. Daros, J.A.; Marcos, J.F.; Hernandez, C.; Flores, R. Replication of avocado sunblotch viroid: evidence for a symmetric pathway with two rolling circles and hammerhead ribozyme processing. Proceedings of the National Academy of Sciences 1994, 91, 12813-12817. doi:10.1073/pnas.91.26.12813.

14. Flores, R.; Daròs, J.; Hernández, C. Avsunviroidae family: viroids containing hammerhead ribozymes. Adv Virus Res 2000, 55, 271-323.

15. Flores, R.; Gas, M.E.; Molina-Serrano, D.; Nohales, M.Á.; Carbonell, A.; Gago, S.; la Peña, M.D.; Daròs, J.A. Viroid Replication: Rolling-Circles Enzymes and Ribozymes. Viruses 2009, 1, 317-334. doi:10.3390/v1020317.

16. Canny, M.D.; Jucker, F.M.; Pardi, A. Efficient Ligation of theSchistosomaHammerhead Ribozyme†. Biochemistry 2007, 46, 3826-3834. doi:10.1021/bi062077r.

17. Hutchins, C.; Keese, P.; Visvader, J.; Rathjen, P.; McInnes, J.; Symons, R. Comparison of multimeric plus and minus forms of viroids and virusoids. Plant Mol Biol 1985, 4, 293-304.

18. Riesner, D.; Steger, G. Temperature-Gradient Gel Electrophoresis of RNA. In Handbook of RNA Biochemistry; Wiley-VCH Verlag GmbH \& Co. KGaA, 2014; pp. 427-444. doi:10.1002/9783527647064.ch21.

19. Delan-Forino, C.; Deforges, J.; Benard, L.; Sargueil, B.; Maurel, M.; Torchet, C. Structural analyses of Avocado sunblotch viroid reveal differences in the folding of plus and minus RNA strands. Viruses 2014, $6,489-506$.

20. Zaccai, N.R.; Serdyuk, I.N.; Zaccai, J. Methods in Molecular Biophysics; Cambridge University Press, 2017. doi:10.1017/9781107297227.

21. Bernadó, P.; Shimizu, N.; Zaccai, G.; Kamikubo, H.; Sugiyama, M. Solution scattering approaches to dynamical ordering in biomolecular systems. Biochim Biophys Acta Gen Subj 2018, 1862, 253-274.

22. Li, Y.; Maurel, M.; Ebel, C.; Vergne, J.; Pipich, V.; Zaccai, G. Self-association of adenine-dependent hairpin ribozymes. Eur Biophys J 2008, 37, 173-82.

23. Leclerc, F.; Zaccai, G.; Vergne, J.; Řìhovà, M.; Martel, A.; Maurel, M.C. Self-assembly Controls Self-cleavage of HHR from ASBVd (-): a Combined SANS and Modeling Study. Scientific Reports 2016, 6. doi:10.1038/srep30287.

24. Delan-Forino, C.; Maurel, M.C.; Torchet, C. Replication of Avocado Sunblotch Viroid in the Yeast Saccharomyces cerevisiae. Journal of Virology 2011, 85, 3229-3238. doi:10.1128/jvi.01320-10.

25. Sato, N. Was the evolution of plastid genetic machinery discontinuous? Trends in Plant Science 2001, 6, 151-155. doi:10.1016/s1360-1385(01)01888-x.

26. Latifi, A.; Bernard, C. Replication of Avocado Sunblotch Viroid in the Cyanobacterium Nostoc Sp. PCC 7120. Journal of Plant Pathology \& Microbiology 2016, 07. doi:10.4172/2157-7471.1000341.

27. Diener, T.O. Circular RNAs: relics of precellular evolution? Proceedings of the National Academy of Sciences 1989, 86, 9370-9374. doi:10.1073/pnas.86.23.9370.

28. Horning, D.P.; Joyce, G.F. Amplification of RNA by an RNA polymerase ribozyme. Proceedings of the National Academy of Sciences 2016, 113, 9786-9791. doi:10.1073/pnas.1610103113.

29. Bergman, N.H. The three-dimensional architecture of the class I ligase ribozyme. RNA 2004, 10, $176-184$. doi:10.1261/rna.5177504. 